\title{
Fluid structure around a tilted rectangular cylinder near a solid wall and induced loading
}

\author{
S. Malavasi, E. Zappa \& A. Cigada \\ Politecnico di Milano, Milan, Italy
}

\begin{abstract}
The main goal of this paper is to investigate the effects of an asymmetric confined flow developing around a cylinder with a rectangular cross-section at different attack angles. Previous studies faced this problem analyzing change of the drag and lift force coefficients due to the proximity of a fixed plate parallel to the mean flow and fixed in the vicinity of the cylinder. In this paper an original application of particle image velocimetry technique was used to investigate the mean fluid structures around rectangular cylinders with aspect ratios of 3 and 4 . The kinematics characterization of the flow field has been quantified through the transversal dimension of the wake and the position of the stagnation point on the frontal area of the obstacle. These parameters have been compared with the mean force coefficients on the cylinder. The non-symmetric boundary conditions were obtained in an open chamber wind tunnel, putting a fixed plate parallel to the mean flow at one side of the cylinder; on the other side an almost free flow can be assumed. The comparison between the kinematics parameters of the flow structure and the dynamic coefficients provides useful information to the phenomenon understanding.
\end{abstract}

Keywords: confined flow, rectangular cylinder, flow induced loading.

\section{Introduction}

The paper focuses on providing new insights and knowledge of the mechanisms governing the flow field around a tilted rectangular cylinder near a solid wall, with particular attention to the study of the mean flow around the cylinder.

The problem of fluid dynamic forces acting on a rectangular cylinder was studied by different Authors, such as Naudasher and Wang [3], Norberg [4] and Deniz and Staubli [5] that considered the cylinder immersed in unbounded flow. 
In case of confined flow the net force acting on the cylinder changes significantly. In literature, major attention was paid to the case of symmetric bounded flow; e.g. Okajima et al. [6] highlighted the influence of the blockage on the fluid-dynamic loading. Recent works of our research group investigated the effects of an asymmetric boundary condition placing the cylinder at different elevation above a solid wall $[1,2]$.

According to literature data (see Simiu and Scanlan [7]) a critical condition for cylinder with zero attack angle is represented by the chord-to-width ratio ranging between 2 and 4 ; this is due to the change of the fluid behaviour as a consequence of an increasing flow tendency to re-attach to the cylinder as the chord-to-width ratio increases (see Deniz and Staubli [5]). This tendency is strongly affected by the Reynolds number value, as underlined in Mills et al. [8].

To examine the correlation between the induced excitation and the flow structure in the case of asymmetric boundary conditions, in this paper we use an original application of a particle image velocimetry technique that allows us to investigate the mean fluid structures around rectangular cylinders located in close proximity of a fixed plate parallel to the mean flow (see Figure 1). The kinematics characterization of the flow field will be based on the estimation of the transversal dimension of the wake and the position of the stagnation point on the frontal area of the obstacle. These parameters have been compared with the mean force coefficients on the cylinder.

\section{Experimental set-up}

The experimental set-up used for the present research is primarily composed by an open chamber wind tunnel with a square $(0.9 \mathrm{~m} \times 0.9 \mathrm{~m})$ main section, a bottom plate (providing a solid boundary), a rectangular cylinder (also referred to in the following as "model") mounted on a purpose-built dynamometer used to hold the model at different heights and attack angles and to measure lift and drag forces. Two dynamometric models with rectangular section will be tested in this paper, both with a width "s" of $40 \mathrm{~mm}$ (see Figure 3) and chords "l" of 120 and $160 \mathrm{~mm}$ respectively; the chord-to-width ratios are then 3 and 4. Mean flow velocity is estimated by a Pitot tube.
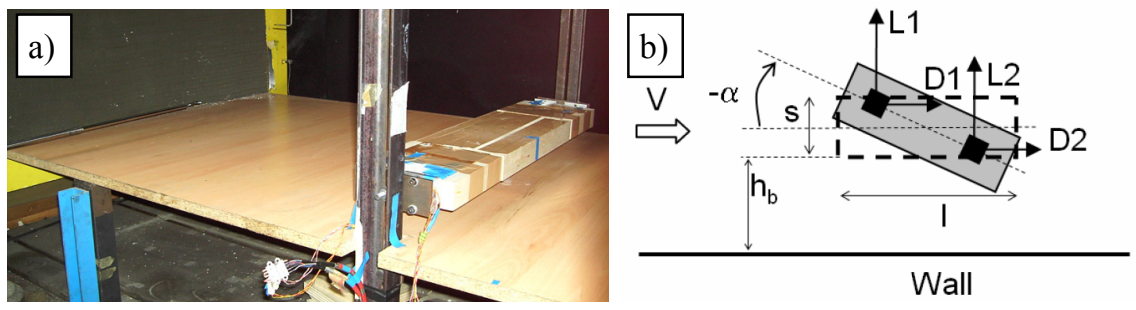

Figure 1: (a) Dynamometric cylinder mounted close to a fixed plate to create non-symmetric boundary conditions; (b) symbols and conventions. 
The boundary layer along the wall was measured in [1] through a hot-wire anemometer and was found to be $40 \mathrm{~mm}$ thick. This means that the model is partially immersed in the boundary layer both in case of tests with $h_{b} / s=1$ and in case of $h_{b} / s>1$ with large attack angle.

The distance between the cylinder and the bottom plate can be changed from $200 \mathrm{~mm}$ (quasi free flow boundary conditions) to $40 \mathrm{~mm}$, (non-symmetric boundary conditions) in presence of non-negligible angles of attack. In the latter condition the fluid-dynamic behaviour of the system is strongly affected by small changes either in geometric or flow conditions [2]. A particle image velocimetry system provides the characterization of the average two-dimensional velocity field around the obstacle, allowing for a better understanding of the flow behaviour at different values of the angle of attack; this information is interesting in particular in case of cylinder very close to the fixed plate. The mean flow field was reconstructed and the position of separation point and the transversal size of the wake were estimated as a function of the attack angle $\alpha$.

In order to study the forces acting on the model in the listed experimental conditions, a dynamometric model is used in the tests; the model is fixed to a sensing element supported by strain-gauge-equipped beams at the two sides. Only the central part of the model is sensitive, while the two sides are dummies; in this way the side effect on the sensing part is kept to a minimum. A detailed description of this sensitive model can be found in [9].

In Figure 1(a) is visible the model, mounted in the wind tunnel, while in Figure 1(b) a side view of the dynamometric model is given, together with the list of symbols. The height of the cylinder, $\mathrm{h}_{\mathrm{b}}$, is measured from the ground to the bottom part of the model. We divided $h_{b}$ by the width "s" of the cylinder to obtain the dimension less parameter $h_{b} / s$. During the tests the $h_{b}$ and $\alpha$ parameters are varied while the lift and drag at both the leading and trailing edges are measured, thanks to the dynamometers that support the model.

For the kinematic characterization of the mean flow field an original particle image velocimetry system, optimized for hydraulic applications (described in [10]) has been adapted for air applications. In the original system the 2D velocity fields are obtained measuring the bi-dimensional trajectories of seeding particles in a defined time interval. This is possible by filming the seeding flow on the measurement plane and by using a delayed exposure time. Since the bubbles tend to spread in the wind tunnel camera, it is necessary to enhance the bubbles belonging to a plane parallel to the mean flow vector and orthogonal to the dynamometric cylinder axis; this is obtained using a lamp able to produce a light plane. The length of the trajectory generated by each bubble is a function of both the instantaneous local flow velocity and to the shutter time of the video-camera. Since the shutter time is imposed (and then known) the flow velocity can be measured starting from the trajectory length, once the system has been calibrated. In the present study we have adapted the particle image velocimetry system mentioned before, using soap bubbles filled with helium to seed the fluid [11], changing the illumination source to obtain a wider light sheet and increasing the acquisition frame rate to allow measurement of speeds an order of magnitude grater than in case of hydraulic application. Due to this kind of 
modifications, it was necessary to change some part of the image processing software, in order to adapt it to the new kind of images, characterized by the presence of a limited number of seeding particle, by a double reflection of the illuminated bubble on the image (both the upper and the lower part of each bubble reflects the light and then each bubble produces 2 lines on the image) and by a significant distance between two consecutive bubble. The soap bubbles used as seeding media are filled with helium; since this gas has a volumetric mass lower than air and then it is possible, by regulating the helium pressure, to compensate the soap weight and obtain bubbles almost unaffected by the gravitational force. The bubbles can then better follow the flow.

The image acquisition is based on a Basler grayscale camera, equipped with a CMOS sensor, characterized by a $656 \times 491$ resolution. The camera is connected to a laptop PC through a FireWire IEEE 1394 bus, used for both the camera configuration and the images acquisition. The software for the acquisition and storage of the images and the force data was developed by the authors using National Instruments LabVIEW. In order to avoid perspective aberration in the grabbed images, the camera was positioned with the optic axis orthogonal to the light plane.

The bubbles, illuminated by a proper light sheet, produce on the images a stripe; by analyzing of each stripe, information concerning the local velocity vector orientation and magnitude can be extracted. First of all the image processing software performs a binarization of all the acquired images, in order to enhance the blobs, representing the bubble stripes; after that each of the blob is analyzed in order to extract the information useful for the subsequent processing (i.e. the centre of mass, the area, the length and orientation and so on). Since no information concerning the velocity direction is given by the blobs, a further analysis has to be done in order to estimate it. The main idea is to analyze the blobs of each image and to compare them with those of the previous and the subsequent ones. If a blob with a certain orientation is present in an image, in the following one we expect to find another blob, having a similar orientation, and positioned at one of the two sides of the first one. Comparing blob positions in subsequent images the software assign the direction of each blob. Averaging in time and in space (on a reference grid) the velocity information we obtained the mean velocity field upstream and downstream the cylinder. The entire flow field reconstruction allowed the determination of significant parameters such as the stagnation point and the wake dimension. The stagnation point on the frontal face of cylinder was estimated by considering the interpolated position of the point with $\mathrm{Vy}=0$ in the velocity vertical distribution upstream the cylinder; the wake dimension by considering the streamline distribution downstream the obstacle.

\section{Uncertainty estimation}

The image acquisition and force measurements are both controlled by two independent acquisition systems. Therefore the two measurement systems are actually independent and the uncertainty should then be estimated separately. 
The force measurement system was calibrated using reference masses with negligible uncertainty. After the calibration, repeated tests were performed applying to the dynamometers known masses; while no significant systematic effects were found, the random effects on lift and drag forces were estimated, and standard deviation of the results was found to be lower than $0.05 \mathrm{~N}$ in all the experimental conditions.

Although only average values of the drag and lift forces will be of interest for this research, natural vibration of the dynamometric cylinder excited by the vortex shedding can lead to inaccurate measurements of the mean forces, due to the reduction of the signal to noise ratio. It is then appropriate to avoid experimental conditions where the vortex shedding frequency is close to one natural frequency of the model. The Strouhal number of a tilted rectangular cylinder near a solid wall was measured in [2]: this parameter can vary between 0.04 and 0.14 in all the experimental conditions to be tested for this research. In the same paper the frequency response function of the dynamometric model mounted in the wind tunnel was estimated and two main resonance peaks were found, one due to the first vertical mode, at $32.6 \mathrm{~Hz}$, and another due to a torsion mode, at $45.5 \mathrm{~Hz}$. Relying on these considerations an average wind speed of $5 \mathrm{~m} / \mathrm{s}$ was selected for all the tests in this research; in this way the vortex shedding frequencies are lower than $20 \mathrm{~Hz}$ and then do not excite the natural frequency of the dynamometric cylinder. An anti-aliasing filter with $45 \mathrm{~Hz}$ cutoff frequency was used for the measurements, while the sampling frequency was set to $150 \mathrm{~Hz}$.

The structure of the flow-filed was estimated through the described particle tracking technique; the uncertainty of these results strongly depends on the uncertainty of the length of the trajectories produced by the bubbles. However it is almost impossible to keep into account the fact that some bubbles, due to velocity components orthogonal to the light plane, go out of the light sheet before the shutter time is finished. This kind of bubbles produces a trajectory shorter than the ones given by the bubbles that remain in the light plane for the whole shutter time. The authors choose then to experimentally estimate the uncertainty linked to the magnitude of the velocity vectors by computing the average value and the standard deviation of the velocity vectors in an undisturbed flow area, obtaining a relatively high uncertainty: the standard deviation is about $15 \%$ of the magnitude. After an average of 600 images, however, the average velocity vector is estimated with an uncertainty much lower (as is obvious) allowing to estimate in a reliable way the position of the stagnation point, as well as the wake size. In order to check whether the 3-second image acquisition reliably represents the average flow field conditions, some test were done: first of all the average flow was estimated though 5 different set of images, each of them constituted by 600 images (3-second record at $200 \mathrm{~Hz}$ grabbing frequency), obtaining a substantial repeatability of the mean flow.

By analysing the dimensionless cumulative average of the horizontal and vertical components of the undisturbed flow velocity, it is possible to see (Figure 2) that the signal tend to become stable (with oscillation of few percentage points) within the 3 -second acquisition. This is a further proof that the average 
over $3 \mathrm{~s}$ interval is representative of the average flow structure interaction. A similar behaviour was found also for the lift and drag forces.

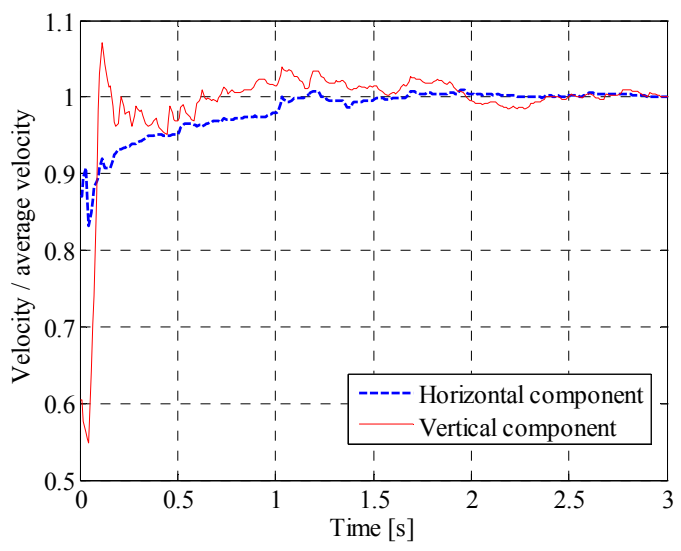

Figure 2: Dimensionless cumulative average of the horizontal and vertical components of the undisturbed flow velocity.

\section{Test results}

For all of the tests the average incoming flow speed was set to $5 \mathrm{~m} / \mathrm{s}$, for the reasons explained in the previous section. The test conditions comprehend low elevation values $\left(\mathrm{h}_{\mathrm{b}} / \mathrm{s}\right.$ ranges from 5 down to 1$)$ and relatively large range of attack angle: $-20^{\circ} \leq \alpha \leq+20^{\circ}$ at high $h_{b} /$ s values, whereas for low elevations the maximum tilting angle is limited by the impact of the model on the fixed plate. In the following of this paper the analysis of the characteristics of the flow field around the model, will be of great help to investigate the causes that produce the drag and lift coefficients behaviour, already discussed in Cigada et al. [1] and in Malavasi and Zappa [2]. As an example in Figure 3 the average flow field around the obstacle is shown in case of zero and $-10^{\circ}$ attack angle for $h_{b} / s=1$ and $1 / \mathrm{s}=3$.
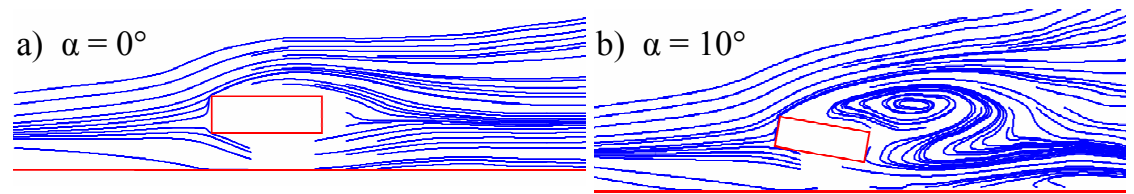

Figure 3: Average flow around the model; $1 / \mathrm{s}=3 ; \mathrm{h}_{\mathrm{b}} / \mathrm{s}=1 ; \mathrm{Re}=1.33 \mathrm{E}+4$.

As can be seen in Figure 3a, the asymmetry of the boundary condition is underlined by the vertical position of the stagnation point, in front of the cylinder. In this case the flow separation point is close to the lower leading edge (the one near the boundary surface); as known both in case of symmetric 
boundary conditions or unbounded flow, the separation point is on the cylinder symmetry axes. The fact that, in case of confined flow the separation point moves towards the confining wall agrees with Malavasi et al. [10], where the rectangular cylinder with shape ration $1 / \mathrm{s}=3$ is studied. Similar results were found by Bosh and Rodi [12] in the case of square section cylinders.

Figure 4 reports the vertical position of the stagnation point divided by the thickness of the cylinder $(\operatorname{Pr} / \mathrm{s})$ vs. $\alpha$ for two aspect ratios $(1 / \mathrm{s}=4$ and 3$)$ and for two elevation of the cylinder above the solid wall $\left(\mathrm{h}_{\mathrm{b}} / \mathrm{s}=5\right.$ and 1$)$.

Here we can note that the effect of the attack angle on the position of the stagnation point is emphasised by the presence of the solid boundary, while it is not significantly affected by the changing of $1 / \mathrm{s}$.
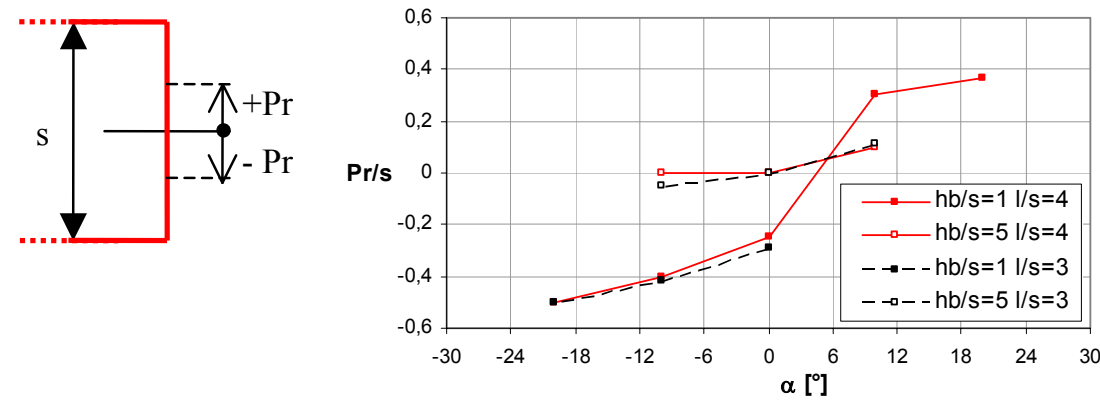

Figure 4: Normalised stagnation point position $\mathrm{Pr} / \mathrm{s}$ as a function of the attack angle $\left(1 / \mathrm{s}=3\right.$ and $4 ; \mathrm{h}_{\mathrm{b}} / \mathrm{s}=1$ and $\left.5 ; \mathrm{Re}=1.33 \mathrm{E}+4\right)$.

As a matter of fact, the increasing of $\mathrm{Pr} / \mathrm{s}$ in the range $-10^{\circ} \leq \alpha \leq 10^{\circ}$, is significantly larger for $h_{b} / s=1$ than for $h_{b} / s=5$. Analysing the downstream flow field shown in Figure $3 \mathrm{a}$ it is possible to note that the flow tends to reattach to the cylinder at the upper and at the lower sides, and then separates again at the trailing edge.

Figure 5 reports the transversal size of the wake divided by the thickness of the cylinder (Ds/s) vs. $\alpha$. The presence of the solid boundary at $\alpha=0$ does not affect significantly the Ds/s ratio; nevertheless, as in the case of $\mathrm{Pr} / \mathrm{s}$, the presence of the confining plate generated a non-symmetric effect of positive and negative values of $\alpha$ on the wake dimension. With the goal of comparing the flow characteristics with the load induced on the cylinder, the average drag and lift coefficients were also measured using the same experimental conditions. Figure 6 and 7 show these parameters as a function of $\alpha$. The lift coefficient values at zero attack angle agree with the experimental observation published by Cigada et al. [1], who found, in experimental conditions analogous to the ones studied in this paper, a significant modification in the lift coefficient, with respect to the case of unbounded flow conditions. The cited paper shows that the drag coefficient, on the contrary, does not vary significantly between bounded and unbounded flow conditions (always at $\alpha=0$ ); this agrees with the fact that the wake dimension does not change significantly with the presence of the solid wall (see Figure 5 at zero attack angle). 
Observing the $\operatorname{Pr} / \mathrm{s}$ parameter and the lift coefficient at $\alpha \neq 0$ it is possible to note that, at $h_{b} / s=5$ both the Pr/s and the $C_{L}$ curves (Figures 4 and 6) show trends with a centrosimmetry, with centre of symmetry at the origin of the coordinates system. On the contrary, when $h_{b} / s=1$ the presence of the confining plate increases the asymmetry of the phenomenon with respect of the attack angle.
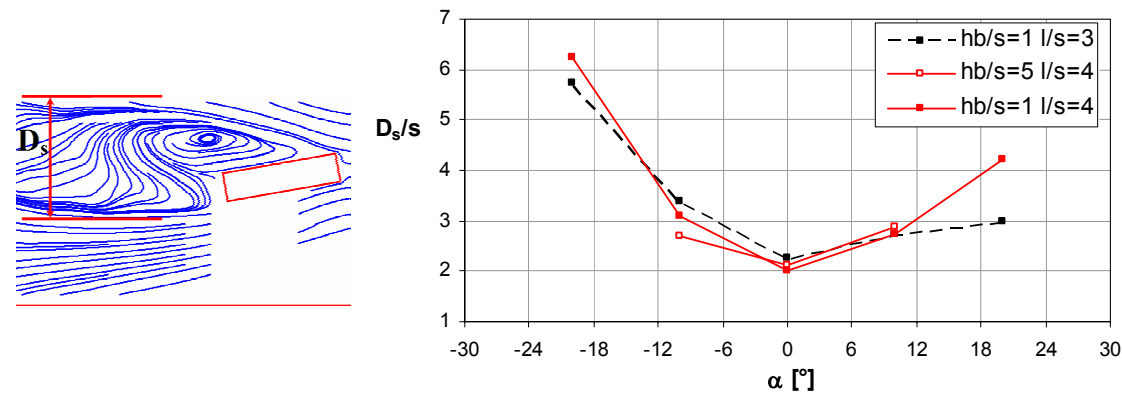

Figure 5: $\quad$ Normalised wake size Ds/s as a function of the attack angle $(1 / \mathrm{s}=3$ and $4 ; \mathrm{h}_{\mathrm{b}} / \mathrm{s}=1$ and $\left.5 ; \mathrm{Re}=1.33 \mathrm{E}+4\right)$.

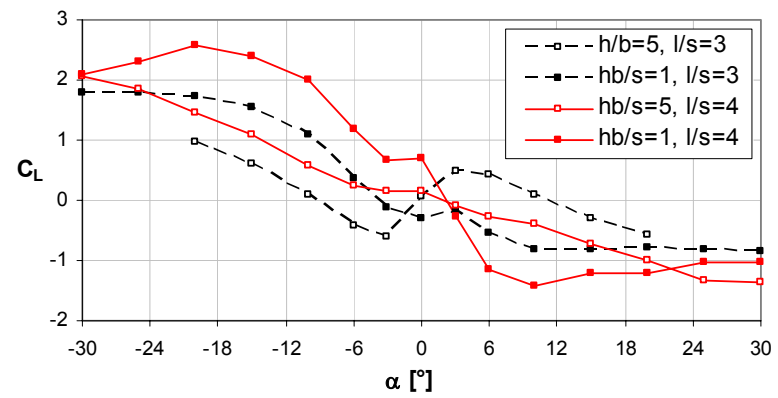

Figure 6: Lift coefficient as a function of the attack angle $(1 / \mathrm{s}=3$ and 4; $\mathrm{h}_{\mathrm{b}} / \mathrm{s}=1$ and $\left.5 ; \mathrm{Re}=1.33 \mathrm{E}+4\right)$.

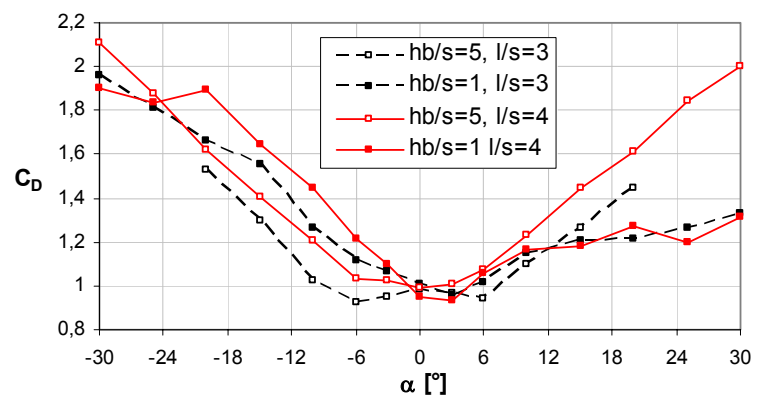

Figure 7: $\quad$ Drag coefficient as a function of the attack angle $(1 / \mathrm{s}=3$ and 4; $\mathrm{h}_{\mathrm{b}} / \mathrm{s}=1$ and $\left.5 ; \mathrm{Re}=1.33 \mathrm{E}+4\right)$. 
Considering the Ds/s and $\mathrm{C}_{\mathrm{D}}$ diagrams it is possible to note that the curves are almost symmetric with respect to the axis of ordinates in case of almost unbounded flow field (Figures 5 and 7 at $h_{b} / s=5$ ). Similarly to what observed for the $\operatorname{Pr} / \mathrm{s}$ and $\mathrm{C}_{\mathrm{L}}$ parameters, also the $\mathrm{D}_{\mathrm{s}} / \mathrm{s}$ and the $\mathrm{C}_{\mathrm{D}}$ curves loose the symmetry for $h_{b} / s=1$ due to the presence of the fixed confining plate. In particular the drag coefficient increases rapidly for negative attack angle values, while the increasing is lower for positive attack angles; the trend of the Ds/s curve is extremely similar to the drag curve trend. The latter founding agrees with evidence by Da Matha et al. [13] in unbounded flow.

To summarise the obtained results it is possible to asses that the confining surface makes the phenomenon non symmetric (this fact is known in literature) and, when the attack angle $\alpha$ is non zero (Figure $4 b$ ), a further asymmetry is introduced in the problem, modifying again the flow around the cylinder; as a consequence of this fact the drag and lift coefficients show significant variations. In this scenario, however, the flow-field characteristics reported in Figure 4 and 5 are consistent with the observation of the force coefficients in the same boundary conditions (Figure 6 and 7). As a matter of fact the trend of the wake size curve is similar to the drag coefficient curve and the position of the separation point is closely related with the lift coefficient, also in case of confined flow and attack angle different from zero.

\section{Conclusion}

In order to investigate the effects on a cylinder with rectangular cross-section placed in an asymmetric confined flow with different attack angles, synergic use of particle tracking technique and direct force measurements was used. The significant experimental condition characterized by low elevation of the cylinder by a solid wall and non-negligible angles of attack are especially considered.

Under these boundary conditions a small change in the tilt value causes an edge of the cylinder to be positioned very close to the wall, thus: the average flow field is substantially different from the other scenarios and the net force acting on the cylinder dramatically changes. The observation of the flow behaviour developing under these conditions contributed to the comprehension the results of the force measurements, providing useful information to the phenomenon understanding, in particular the position of the stagnation point was found to be strictly related to the lift while the size of the wake significantly affects the drag coefficient, not only in case of zero attack angle but also in case of arbitrary values of $\alpha$.

\section{Acknowledgements}

The work was supported by the Italian Ministero dell'Università e della Ricerca Scientifica e Tecnologica (PRIN 2005 - research title: "Ricerca di base sulle vibrazioni indotte da fluidi su strutture flessibili"). The authors thank Giuseppe Crosta for the technical support concerning the bubble generator and Orlando Vallerio for his contribution for the colleting of the experimental data. 


\section{References}

[1] Cigada A., Malavasi S. \& Vanali M. 2006 Effects of an asymmetrical confined flow on a rectangular cylinder. Journal of Fluid and Structure, 22 (2), pp.213-227.

[2] Malavasi, S \& Zappa, E. 2006, Fluid-Dynamic Loading On A Tilted Rectangular Cylinder Near A Solid Wall. Proceedings of PVP2006ICPVT-11 2006 ASME Pressure Vessels and Piping Division Conference, July 23-27, 2006, Vancouver, BC, Canada.

[3] Naudasher E. \& Wang Y. 1993 Flow-induced vibrations of prismatic bodies and grids of prisms. Journal of Fluids and Structures $n^{\circ} 7$, pp. 341-373.

[4] Norberg, C. 1993 Flow around rectangular cylinders, pressure forces and wake frequencies. Journal of Wind Engineering and Industrial Aerodynamics, vol. 49, pp.187-196

[5] Deniz, S. \& Staubli, Th. 1997 Oscillating rectangular and octagonal profiles: integration of leading- and trailing-edge vortex formation. Journal of Fluids and Structures $n^{\circ} 11(1)$, pp. 3-31.

[6] Okajima, A., Yi, D., Kimura, S. \& Kiwata, T., 1997. The blockage effects for an oscillating rectangular cylinder at moderate Reynolds number. Journal of Wind Engineering and Industrial Aerodynamics 69-71, 997-1011.

[7] Simiu, E. \& Scanlan, R.H. 1996, Wind effects on structures, John Wiley \& Sons.

[8] Mills, R., Sheridan, J. \& Hourigan, K. Particle image velocimetry and visualization of natural and forced flow around rectangular cylinders, Journal of Fluid Mechanics (2003), vol. 478, pp. 299-323.

[9] Cigada A., Falco M. \& Zasso A. 2001 "Development o new systems to measure the aerodynamic forces on section models in wind tunnel testing" Journal of wind engineering and industrial aerodynamics Vol 89/7-8, pp 725-746.

[10] Malavasi S. Franzetti S. \& Blois G. PIV investigation of flow around submerged river bridge deck, River Flow 2004, $2^{\text {nd }}$ International Conference of Fluvial Hydraulics, June 2004, Napoli-Italy, pp. 601-608.

[11] De Ponte, S., Svelto C., Malavasi S. \& Franzetti S. Improvement in the 3color Particle Image Velocimetry System, IMTC 2004, Como - Italy, 18 20 May 2004, pp. 1053-1057.

[12] Bosch, Rodi 1996 Simulation of vortex shedding past a square cylinder near a wall, International Journal of Heat and Fluid Flow 17, pp. 267-275.

[13] Da Matha F.A., Laneville A., Trépanier J.Y. \& Lü Z.Y. 1988 Detailed pressure field measurements for some 2-D rectangular cylinders. Journal of Wind Engineering and Industrial Aerodynamics, vol. 28, pp.241-250.

[14] Yu, D. \& Kareem, A. 1998 "Parametric study of flow around rectangular prisms using LES" Journal of wind Engineering and Industrial Aerodynamics vol. 77\&78, pp. 653-662. 